\title{
Insilico Analysis of Protein-Ligand Docking of DHFR (Dihydro Folate Reductase) and Quassinoids
}

\author{
R. D. Shailima Vardhini \\ Head of the Department, \\ Biochemistry, \\ St. Marys College, \\ Yousufguda, \\ Hyderabad.
}

\begin{abstract}
Quassinoids are the naturally available plant extracts which exhibit a wide range of biological activities, that include anti malarial, anti amoebic, anti tumor properties etc. The present experiment aims to exploit the antitumor properties of the quassinoids. 68 quassinoid analogues were designed and were docked with hDHFR (human dihydrofolate reductase) a potential cancer target. The docking results showed compound 33 to be the best ligand for DHFR.
\end{abstract}

\section{KEYWORDS}

Quassinoids, quassinoid ligands, quassinoids analogues, anti tumor, DHFR.

\section{INTRODUCTION:}

Quassinoids are the naturally available plant agents which exhibit a host of biological activities [1,2], seen mostly in Simaroubaceae species [3]. These biologically active phytochemical agents belong to the triterpene chemical family. The main active groups of the Quassinoids are Ailanthionone, Glaucorubinone and Holacanthone besides Benzoquinone, Canthin, Dehydroglaucarubinone and also Glaucarubine, Simarolide, Sitosole and Melianone [4].

The picrasane skeleton, a pentacyclic derivative of the Quassinoids have shown a remarkable antitumor activity [5]. Quassinoids also are known for their potent antimalarial [6], antimicrobial and antiprotozoal activities $[7,8]$.

The present experiment aims to create the analogues for Quassinoids exploiting their antitumor property and study the binding affinity of these analogues for hDHFR, a target for Cancer [9].

Fluoropyrimidine - Antifolate based chemotherapeutic are extensively used to decrease the number of tumors.Human DHFR (hDHFR) Dihydrofolate reductase EC 1.5.1.3 is an enzyme with 186 amino acids and bears the molecular weight of $21.3 \mathrm{k} \mathrm{Da}$. The gene coding the human DHFR is found in q11q22 region of the chromosome 5 [10].
DHFR catalyses the reduction of 7,8 dihydro folate to folate 5,6,7,8-tetrahydrofolate (THF) which is an NADPH dependent reduction. THF are needed for a majority of one-carbon transfer reaction, in the biosynthesis of serine, glycine, thymidylate, purine, pyrimidine synthesis etc.

DHFR is useful in maintaining the intracellular folate pool and its depletion results in depletion of folate pool and hence effects the one-carbon transfer reactions.[11]

\section{MATERIALS AND METHODS:}

\subsection{Ligands Preparation:}

The quassinoid acts as a ligand for the present study and were drawn using chemsketch (ACDLABS 12.0). Removal of duplicates were done and bonds were then added to it. The CHARM $\mathrm{m}$ force field was used to minimize the energy and thereafter using catalyst the $3 \mathrm{D}$ structures were generate

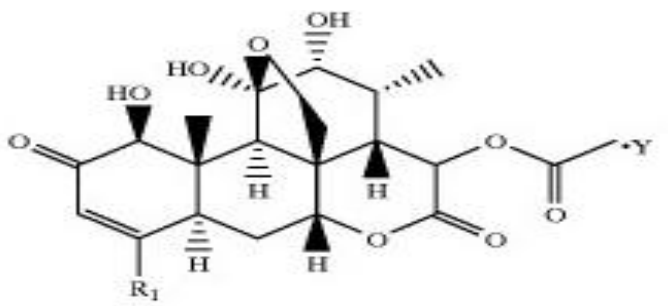

Figure 1: Structure of Quassinoid.

Further, 68 ligands were designed by substituting the groups at $R_{1}$ position and $Y$ position [12]. This was followed by the ligand optimization. 
Table 1. Substitution at $R_{1}$ and $Y$ positions.

\begin{tabular}{|c|c|c|}
\hline Compound & $\mathrm{R}_{1}$ & $\mathrm{Y}$ \\
\hline 1 & $\mathrm{H}$ & $\mathrm{CH}_{3}$ \\
\hline 2 & $\mathrm{H}$ & $\mathrm{C}_{6} \mathrm{H}_{5}$ \\
\hline 3 & $\mathrm{H}$ & $\mathrm{COOH}$ \\
\hline 4 & $\mathrm{H}$ & CYCLOPROPYL \\
\hline 5 & $\mathrm{CH}_{3}$ & $\mathrm{CH}_{3}$ \\
\hline 6 & $\mathrm{CH}_{3}$ & $\mathrm{C}_{6} \mathrm{H}_{5}$ \\
\hline 7 & $\mathrm{CH}_{3}$ & $\mathrm{COOH}$ \\
\hline 8 & $\mathrm{CH}_{3}$ & CYCLOPROPYL \\
\hline 9 & $\mathrm{~F}$ & $\mathrm{CH}_{3}$ \\
\hline 10 & $\mathrm{~F}$ & $\mathrm{C}_{6} \mathrm{H}_{5}$ \\
\hline 11 & $\mathrm{~F}$ & $\mathrm{COOH}$ \\
\hline 12 & $\mathrm{~F}$ & CYCLOPROPYL \\
\hline 13 & ARYL & $\mathrm{CH}_{3}$ \\
\hline 14 & ARYL & $\mathrm{C}_{6} \mathrm{H}_{5}$ \\
\hline 15 & ARYL & $\mathrm{COOH}$ \\
\hline 16 & ARYL & CYCLOPROPYL \\
\hline 17 & SULFO & $\mathrm{CH}_{3}$ \\
\hline 18 & SULFO & $\mathrm{Ph}$ \\
\hline 19 & SULFO & $\mathrm{COOH}$ \\
\hline 20 & SULFO & CYCLOPROPYL \\
\hline 21 & NITRO & $\mathrm{CH}_{3}$ \\
\hline 22 & NITRO & $\mathrm{Ph}$ \\
\hline 23 & NITRO & $\mathrm{COOH}$ \\
\hline 24 & NITRO & CYCLOPROPYL \\
\hline 25 & CARBOXYL & $\mathrm{CH}_{3}$ \\
\hline 26 & CARBOXYL & $\mathrm{Ph}$ \\
\hline 27 & CARBOXYL & $\mathrm{COOH}$ \\
\hline 28 & CARBOXYL & CYCLOPROPYL \\
\hline 29 & $\mathrm{OH}$ & $\mathrm{CH}_{3}$ \\
\hline 30 & $\mathrm{OH}$ & $\mathrm{Ph}$ \\
\hline 31 & $\mathrm{OH}$ & $\mathrm{COOH}$ \\
\hline 32 & $\mathrm{OH}$ & CYCLOPROPYL \\
\hline 33 & $\mathrm{H}$ & $\mathrm{H}$ \\
\hline 34 & $\mathrm{OMe}$ & $\mathrm{CH}_{3}$ \\
\hline 35 & $\mathrm{OMe}$ & $\mathrm{Ph}$ \\
\hline 36 & $\mathrm{OMe}$ & $\mathrm{COOH}$ \\
\hline 37 & $\mathrm{OMe}$ & CYCLOPROPYL \\
\hline 38 & $\mathrm{OMe}$ & $\mathrm{H}$ \\
\hline 39 & Nitro & $\mathrm{H}$ \\
\hline 40 & Sulfo & $\mathrm{H}$ \\
\hline 41 & Aryl & $\mathrm{H}$ \\
\hline 42 & F & $\mathrm{H}$ \\
\hline 43 & $\mathrm{CH}_{3}$ & $\mathrm{H}$ \\
\hline
\end{tabular}

If $\mathrm{Y}$ side chain is referred by the formula [11] 
Table 2. Substitution at $Y$ positions for $R_{2}, R_{3}, R_{4}$.

\begin{tabular}{|c|c|c|c|c|}
\hline COMPOUND & R1 & $\mathrm{R} 2$ & R3 & R4 \\
\hline 44 & $\mathrm{H}$ & $\mathrm{CH}_{3}$ & $\mathrm{CH}_{3}$ & $\mathrm{CH}_{3}$ \\
\hline 45 & $\mathrm{OCH}_{3}$ & $\mathrm{CH}_{3}$ & $\mathrm{CH}_{3}$ & $\mathrm{CH}_{3}$ \\
\hline 46 & ARYL & $\mathrm{CH}_{3}$ & $\mathrm{CH}_{3}$ & $\mathrm{CH}_{3}$ \\
\hline 47 & SULFO & $\mathrm{CH}_{3}$ & $\mathrm{CH}_{3}$ & $\mathrm{CH}_{3}$ \\
\hline 48 & NITRO & $\mathrm{CH}_{3}$ & $\mathrm{CH}_{3}$ & $\mathrm{CH}_{3}$ \\
\hline 49 & $\mathrm{~F}$ & $\mathrm{CH}_{3}$ & $\mathrm{CH}_{3}$ & $\mathrm{CH}_{3}$ \\
\hline 50 & $\mathrm{CH}_{3}$ & $\mathrm{CH}_{3}$ & $\mathrm{CH}_{3}$ & $\mathrm{CH}_{3}$ \\
\hline 51 & CARBOXYL & $\mathrm{CH}_{3}$ & $\mathrm{CH}_{3}$ & $\mathrm{CH}_{3}$ \\
\hline 52 & $\mathrm{H}$ & $\mathrm{CH}_{3}$ & $\mathrm{CH}_{3}$ & $\mathrm{OH}$ \\
\hline 53 & $\mathrm{OCH}_{3}$ & $\mathrm{CH}_{3}$ & $\mathrm{CH}_{3}$ & $\mathrm{OH}$ \\
\hline 54 & ARYL & $\mathrm{CH}_{3}$ & $\mathrm{CH}_{3}$ & $\mathrm{OH}$ \\
\hline 55 & SULFO & $\mathrm{CH}_{3}$ & $\mathrm{CH}_{3}$ & $\mathrm{OH}$ \\
\hline 56 & NITRO & $\mathrm{CH}_{3}$ & $\mathrm{CH}_{3}$ & $\mathrm{OH}$ \\
\hline 57 & $\mathrm{~F}$ & $\mathrm{CH}_{3}$ & $\mathrm{CH}_{3}$ & $\mathrm{OH}$ \\
\hline 58 & $\mathrm{CH}_{3}$ & $\mathrm{CH}_{3}$ & $\mathrm{CH}_{3}$ & $\mathrm{OH}$ \\
\hline 59 & CARBOXYL & $\mathrm{CH}_{3}$ & $\mathrm{CH}_{3}$ & $\mathrm{OH}$ \\
\hline 60 & $\mathrm{H}$ & $\mathrm{C}_{2} \mathrm{H}_{5}$ & $\mathrm{OH}$ & $\mathrm{C}_{2} \mathrm{H}_{5}$ \\
\hline 61 & $\mathrm{OMe}$ & $\mathrm{C}_{2} \mathrm{H}_{5}$ & $\mathrm{OH}$ & $\mathrm{C}_{2} \mathrm{H}_{5}$ \\
\hline 62 & ARYL & $\mathrm{C}_{2} \mathrm{H}_{5}$ & $\mathrm{OH}$ & $\mathrm{C}_{2} \mathrm{H}_{5}$ \\
\hline 63 & SULFO & $\mathrm{C}_{2} \mathrm{H}_{5}$ & $\mathrm{OH}$ & $\mathrm{C}_{2} \mathrm{H}_{5}$ \\
\hline 64 & SULFO & $\mathrm{C}_{2} \mathrm{H}_{5}$ & $\mathrm{OH}$ & $\mathrm{C}_{2} \mathrm{H}_{5}$ \\
\hline 65 & NITRO & $\mathrm{C}_{2} \mathrm{H}_{5}$ & $\mathrm{OH}$ & $\mathrm{C}_{2} \mathrm{H}_{5}$ \\
\hline 66 & $\mathrm{~F}$ & $\mathrm{C}_{2} \mathrm{H}_{5}$ & $\mathrm{OH}$ & $\mathrm{C}_{2} \mathrm{H}_{5}$ \\
\hline 67 & $\mathrm{CH}_{3}$ & $\mathrm{C}_{2} \mathrm{H}_{5}$ & $\mathrm{OH}$ & $\mathrm{C}_{2} \mathrm{H}_{5}$ \\
\hline 68 & $\mathrm{COOH}$ & $\mathrm{C}_{2} \mathrm{H}_{5}$ & $\mathrm{OH}$ & $\mathrm{C}_{2} \mathrm{H}_{5}$ \\
\hline
\end{tabular}

\subsection{Protein Preparation:}

The protein for the present study is imported from PDB [ Protein Data Bank]. The X-Ray crystal structure with high resolution of human DHFR (Dihydrofolate reductase) PDB ID: 1KMS was imported into the discovery studio (Accelrys 2.1).

The protein chemistry of the missing hydrogen was corrected after which the hetero atoms and the crystallographic water

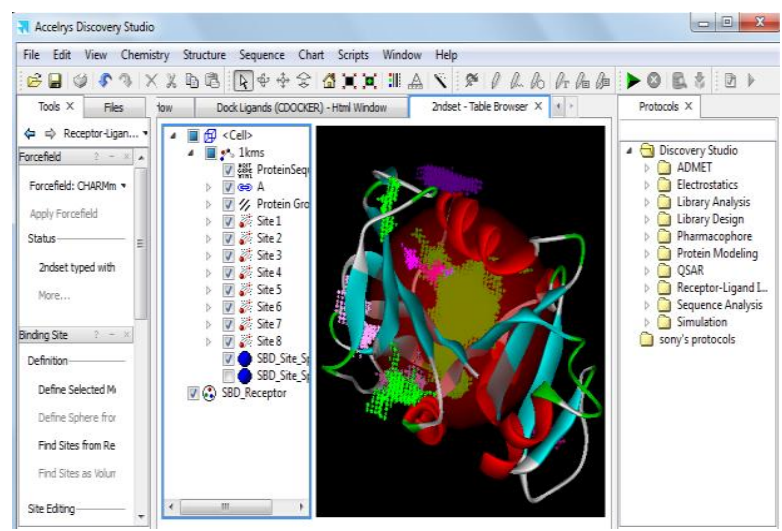

Figure 2. Active site pockets by Eraser algorithm molecules were removed from the protein. Valence monitor options and alternate conformations were used to connect the crystallographic disorder and the unfilled valence atoms. The protein was then subjected to energy minimization steps by applying the steepest descent method which was then followed then followed by the conjugate gradient method until the convergence gradient was satisfied. The active site pockets of the protein hDHFR were identified using eraser algorithm and a sphere was created around the active site.

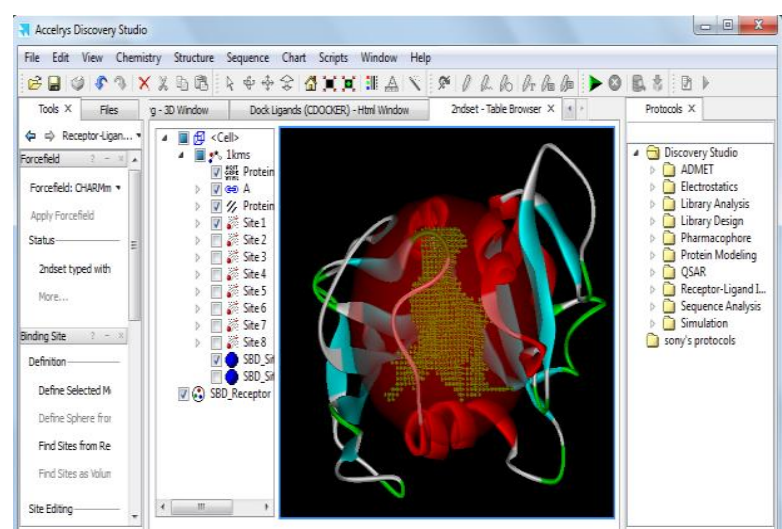

Figure 3. Sphere around the active site pocket 


\subsection{Ligand - Protein Docking:}

Protein - Ligand docking is a molecular modeling technique aims at predicting the position and orientation of the ligand when it binds to the proteins. This method is mostly employed in designing the new drugs. A CHARMm-based docking program DOCK algorithm which offers a full ligand flexibility (including bonds, angles, dihedrals) was employed to find the potential binding mode between both protein and the ligand. In the present

experiment the Quassiniod analogues were docked with the hDHFR and the binding affinity was studied.

\section{Results And Discussions:}

The Protein - Ligand interaction of hDHFR and Quassinoids was studied. Compound-33 shows Highest binding affinity$78.591 \mathrm{~K} . \mathrm{cal} / \mathrm{mol}$ and good hydrogen bonding interactions with active site residues of Human DHFR, which shows Hydrogen bonding interactions with Tyr121, Ala9, Phe34, Ile16, and Val 115.

Table 3. Dock results

\begin{tabular}{|c|c|c|c|c|c|c|c|}
\hline Compound & $\begin{array}{c}\text { Lig } \\
\text { Score } 1\end{array}$ & $\begin{array}{c}\text { Lig } \\
\text { Score } 2\end{array}$ & Plp1 & Plp2 & Jain & Pmf & Dock score \\
\hline \multicolumn{8}{|l|}{ Comp 1} \\
\hline Comp 2 & 2.2 & 2.77 & 87.21 & 85.42 & 4.52 & 124.95 & 36.73 \\
\hline Comp 3 & 1.72 & 2.01 & 85.73 & 82.21 & 4.32 & 113.8 & 33.054 \\
\hline Comp 4 & 1.75 & 2.42 & 85.67 & 81.96 & 4.66 & 114.65 & 33.19 \\
\hline Comp10 & 1.6 & 2.5 & 71.39 & 75.1 & 4.12 & 121.83 & 34.321 \\
\hline Comp 11 & 1.73 & 2.44 & 55.09 & 58.31 & 4.73 & 83.28 & 29.543 \\
\hline Comp 12 & 1.83 & 2.82 & 63.43 & 66.48 & 3.98 & 106.15 & 31.539 \\
\hline Comp 13 & 1.51 & 2.53 & 69.84 & 77.22 & 5.73 & 81.37 & 34.417 \\
\hline Comp 14 & 1.79 & 2.96 & 83.63 & 88.86 & 5.86 & 111.11 & 42.248 \\
\hline Comp 15 & 1.41 & 2.23 & 70.24 & 77.17 & 5.28 & 88.98 & 35.831 \\
\hline Comp 16 & 1.77 & 2.86 & 78.2 & 83.29 & 6.16 & 99.67 & 39.013 \\
\hline Comp 17 & 2.36 & 2.78 & 59.93 & 63.28 & 4.3 & 80.48 & 34.668 \\
\hline Comp 18 & 2.9 & 3.29 & 86.32 & 87.15 & 4.82 & 108.74 & 35.66 \\
\hline Comp 19 & 2.52 & 2.6 & 75.68 & 79.48 & 4.3 & 94.51 & 36.636 \\
\hline Comp 20 & 2.23 & 2.66 & 71.01 & 74.97 & 5.41 & 98.58 & 3.649 \\
\hline Comp 21 & 1.35 & 2.47 & 59.12 & 57.36 & 4.27 & 91.56 & 24.52 \\
\hline Comp 22 & 1.34 & 2.84 & 74.78 & 70.95 & 4.68 & 109.32 & 32.143 \\
\hline Comp 23 & 1.21 & 2.37 & 69.35 & 64.92 & 4.41 & 94.85 & 27.259 \\
\hline Comp 24 & 1.37 & 2.81 & 75.11 & 70.84 & 4.73 & 109.41 & 31.651 \\
\hline Comp 30 & 2.33 & 2.45 & 93.49 & 92.6 & 4.3 & 124.97 & 36.971 \\
\hline Comp 31 & $3 . .1$ & 2.38 & 80.71 & 79.53 & 4.52 & 90.44 & 34.581 \\
\hline Comp 32 & 1.76 & 2.94 & 75.59 & 76.14 & 3.6 & 117.79 & 34.363 \\
\hline
\end{tabular}




\begin{tabular}{|l|l|l|l|l|l|l|l|}
\hline Comp 33 & 2.53 & 3.82 & 56.7 & 58.68 & 4.13 & 116.05 & 45.855 \\
\hline Comp 34 & 1.63 & 2.86 & 64.08 & 63.36 & 5.15 & 88.26 & 33.754 \\
\hline Comp 35 & 1.84 & 3.39 & 82.97 & 81.35 & 5.29 & 101.15 & 42.721 \\
\hline Comp 36 & 1.83 & 2.81 & 70.55 & 69.33 & 4.62 & 87.29 & 36.224 \\
\hline Comp 37 & 1.86 & 3.15 & 79.85 & 73.79 & 3.67 & 115.49 & 39.101 \\
\hline Comp 38 & 1.61 & 3.44 & 56.87 & 56.43 & 3.74 & 101.42 & 40.981 \\
\hline Comp 39 & 2.46 & 3.16 & 69.16 & 68.09 & 4.16 & 114.71 & 37.346 \\
\hline Comp 40 & 2.41 & 3.09 & 85.16 & 80.27 & 3.55 & 99.75 & 36.392 \\
\hline Comp 41 & 1.51 & 2.97 & 62.39 & 64.75 & 5.18 & 95.34 & 39.423 \\
\hline Comp 42 & 2.36 & 3.42 & 54.43 & 60.39 & 4.75 & 109.06 & 42.487 \\
\hline Comp 43 & 2.2 & 3.81 & 81.8 & 79.47 & 4.32 & 127.25 & 42.182 \\
\hline Comp 44 & 2.06 & 2.94 & 82.61 & 83.56 & 4.23 & 121.74 & 36.481 \\
\hline Comp 53 & 1.21 & 2.01 & 70.92 & 80.63 & 6.45 & 97.75 & 26.188 \\
\hline
\end{tabular}

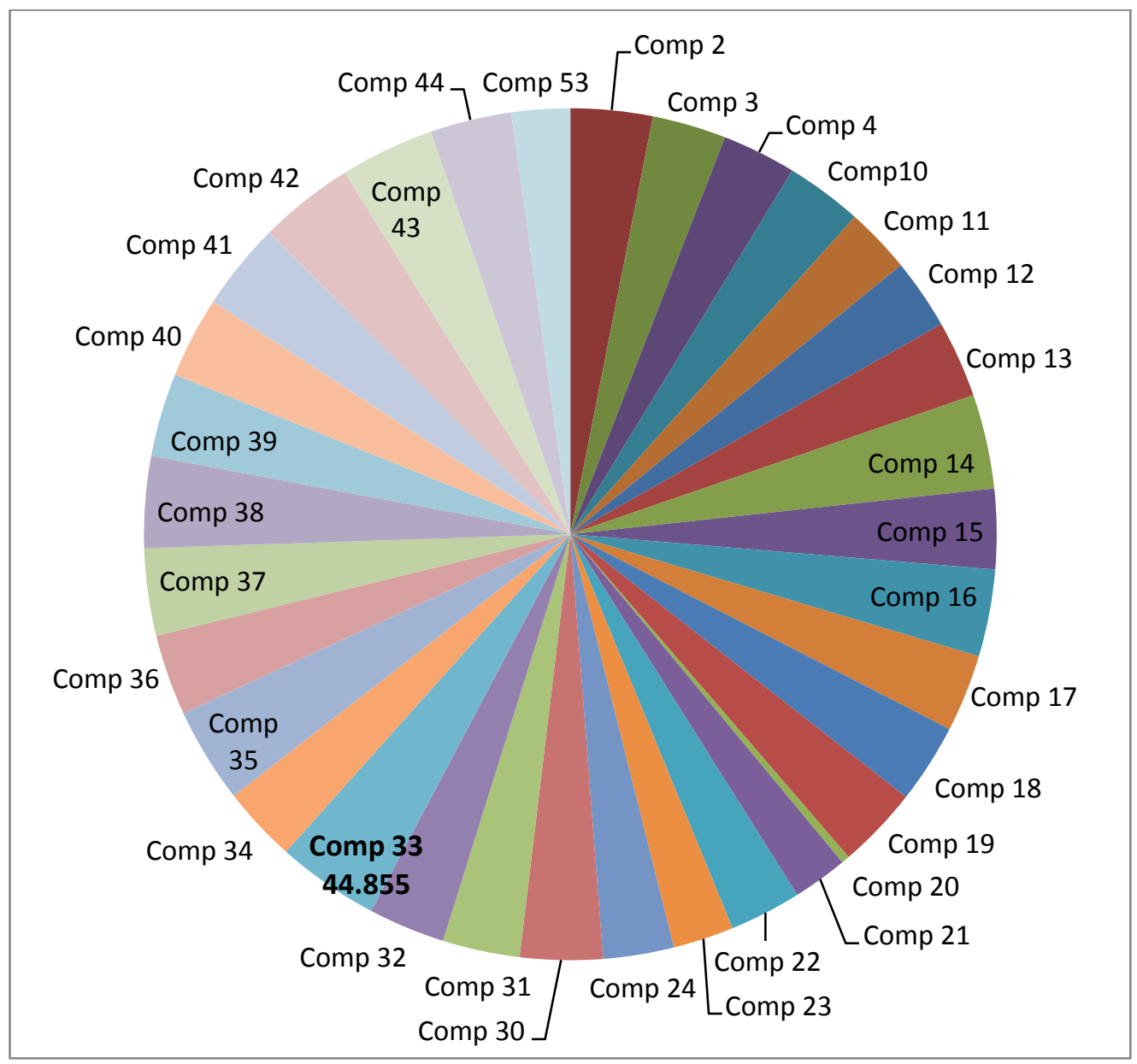

Figure 4. Graphical representation of the Dock results. 


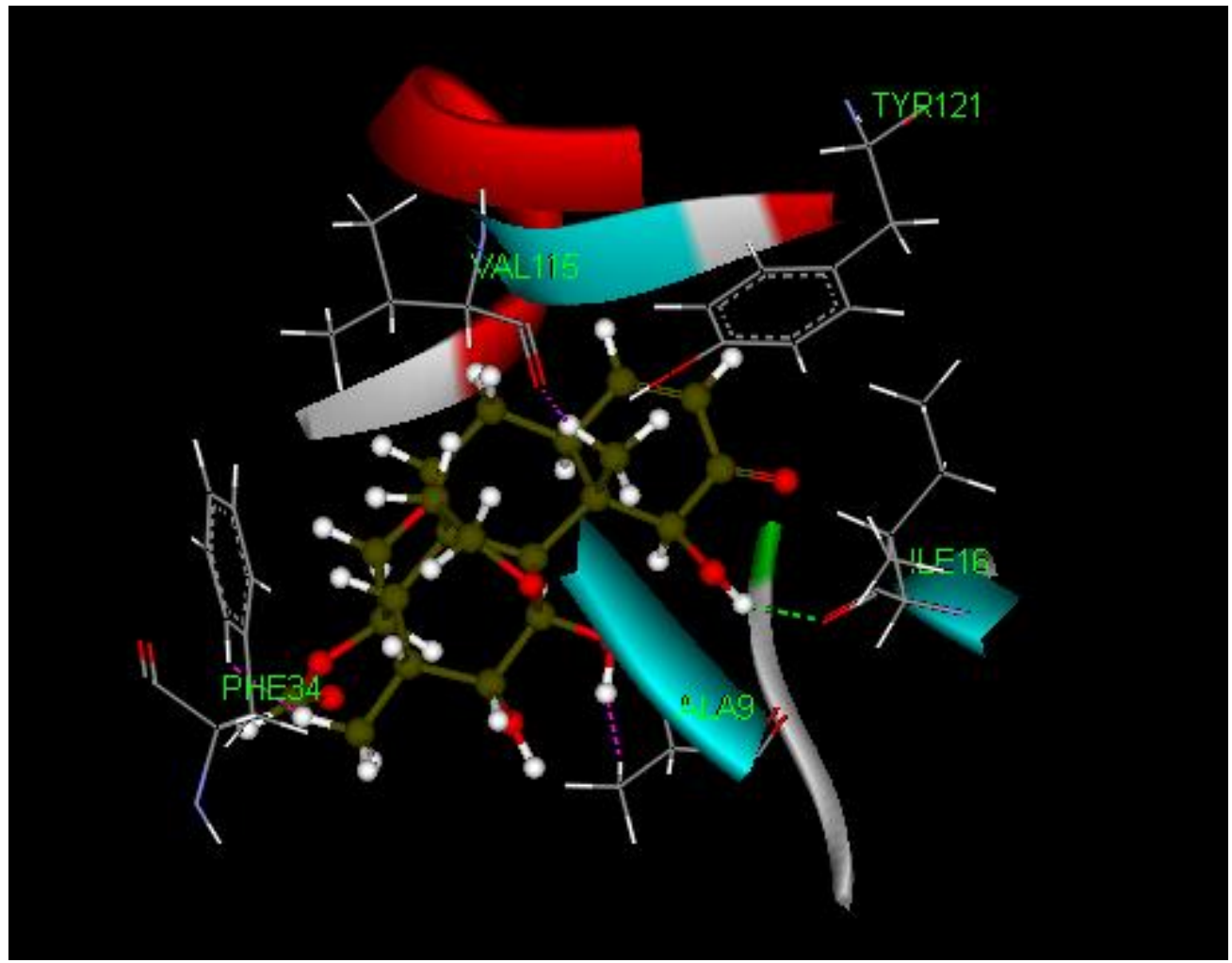

Figure 4. Docking of compound 33 with DFHR

\section{CONCLUSION:}

In the present experiment, the protein-ligand docking studies were performed to explore the binding affinity of hDHFR and the Quassinoids.The study also explores Quassinoids and its analogues to act as an alternative drugs for cancer which acts effectively on hDHFR, a cancer target.

\section{REFERENCES}

[1] Pierre,A

Robert-gero, $\mathrm{M}$

Tempete, $\mathrm{C}$ Polonsky,J.Biochem.Biophys.Res Commun.1980, 93,675.

[2] Spino, C Can.Chem. News 1995,47,16.

[3] Polonsky, J. Fortschr. Chem. Org.Naturst.1973, 30,$101 ; 1985,47,222$

[4] Technical Data Report for Simarauba (Simarouba Amara). Saga Press, Inc 2002.

[5] A.F. Barrero, E. J. Alvarez- Manzaneda, R. AlvarezManzaneda, R. Chahboun, R. Meneses, J.M. Cuerva, M.Aparicio and J.L. Romera, Organic Letters, 2001, vol.3, no,5,647-650.
[6] Melanie J.O'Neill, Dorothy H. Bray, Peter Boardman, J. David Phillipson, David C. Warhurst, Wallance Peters and Matthew Suffness, Antimicrobial Agents and chemotherapy, July 1986, Vol. 30, No .1 P. 101-104.

[7] Wright, C.W., et al . J. Eukaryote. Microbio. 1993;40 (3): 244-6.

[8] Monjour, L. et al , C.R. Acad. Sci. III. 1987; 304 (6): 129 32.

[9] Dihydrofolate Reductase October 2002 Molecule of the Month by David Goodsell/10.2210/rcbs/pdb.

[10] http://www.omicsonline.org/ArchiveJPB/2009/June/03/JPB 2.274.php

[11] Vivek Srivastava et al , Bioinformatics 3(4):180-188 (2008).

[12] Grieco, Morre, Corbett, Valeriote, Feb.14, 2002, United States Patent Application Publication, Pub. No. : US 2002/0019439 